\title{
PREVALENCIA DE ANTICUERPOS A RICKETTSIAS Y EHRLICHIAS EN CUATRO DEPARTAMENTOS FRONTERIZOS DEL PERÚ
}

\author{
Elizabeth Anaya-Ramírez ${ }^{1, a}$, Rosa Palacios-Salvatierra $2, a$, Patricia Mosqueraa,d, Carlos Álvarez ${ }^{3, b}$, \\ Carlos Peralta ${ }^{4, a}$, Rommell Gonzales ${ }^{5, c}$, Silvia Sakuray ${ }^{6, a}$
}

\begin{abstract}
RESUMEN
Para determinar la prevalencia de anticuerpos a Rickettsia y Ehrlichia, relacionada a contacto reciente y pasado, en localidades fronterizas de los departamentos Loreto, Madre de Dios, Tumbes y Tacna, durante los años 2010 y 2011 , se llevaron a cabo encuestas para evaluación serológica empleando el ensayo de inmunofluorescencia indirecta (IFI), a fin de detectar inmunoglobulinas $\mathrm{G}(\mathrm{IgG})$ e inmunoglobulinas totales $(\lg \mathrm{A}+\lg \mathrm{M}+\lg \mathrm{g})$ en 1634 personas. Se encontró una prevalencia de contacto reciente para Ehrlichia de 3,7\% (IC95\%: 3,0-4,4) y para Rickettsia de 10,6\% (IC95\%: 9,112,1), y de contacto pasado para Ehrlichia de 19,0\% (IC95\%: 17,1-21,0) y para Rickettsia de 23,3\% (IC95: 21,2-25,3). Se concluye, que existe una mayor prevalencia para contacto o infección pasada en la población estudiada, tanto para Rickettsia como para Ehrlichia, lo cual nos estaría indicando un comportamiento endémico de ambos agentes infecciosos en las áreas geográficas mencionadas.
\end{abstract}

Palabras clave: Rickettsia; Ehrlichia; Prevalencia; Técnica del anticuerpo fluorescente indirecta; Perú (Fuente: DeCS BIREME).

\section{PREVALENCE OF ANTIBODIES TO RICKETTSIAS AND EHRLICHIAS IN FOUR BORDER DEPARTMENTS OF PERU}

\begin{abstract}
To determine the prevalence of antibodies to Rickettsia and Ehrlichia, related to recent and past contact, in the border towns of the departments Loreto, Madre de Dios, Tumbes and Tacna, during the years 2010 and 2011, surveys for serological evaluation were carried out using the indirect immunofluorescence assay (IIF), in order to detect immunoglobulins G $(\mathrm{lgG})$ and Total immunoglobulins ( $\mathrm{lgA}+\operatorname{lgM}+\operatorname{lgG})$ in 1634 persons. A recent contact prevalence was found for Ehrlichia of 3.7\% (Cl 95\%: 3.0-4.4) and for Rickettsia of 10.6\% (Cl 95\%: 9.1-12.1), and past contact for Ehrlichia of $19.0 \%$ (Cl 95\%: 17.1-21.0) and for Rickettsia of $23.3 \%$ (CI 95\%: 21.2-25.3). It is concluded that there is a higher prevalence for past contact or infection in the population studied, both for Rickettsia and Ehrlichia, which would indicate an endemic behavior to both infectious agents in the geographic areas mentioned.
\end{abstract}

Key words: Rickettsia; Ehrlichia; Prevalence; Fluorescent antibody technique, indirect; Peru (Source: MeSH NLM).

\section{INTRODUCCIÓN}

El síndrome febril indiferenciado es un cuadro clínico frecuente en Perú que, muchas veces queda sin diagnóstico etiológico. La Rickettsia y la Ehrlichia son microorganismos involucrados en la presentación de síndrome febril de carácter inespecífico ${ }^{(1)}$, se presentan en diversos ámbitos geográficos, su circulación y distribución se relacionan con cambios climáticos que aceleran los ciclos biológicos de los vectores, han sido causantes de numerosas epidemias en diversos grupos poblacionales ${ }^{(3,4)} \mathrm{y}$, además, persisten en entornos epidemiológicos definidos, con presencia de artrópodos vectores y animales domésticos como reservorios ${ }^{(2)}$.

En diversas latitudes del planeta se han realizado estudios seroepidemiológicos para elucidar las características de su presencia y circulación ${ }^{(5)}$. En

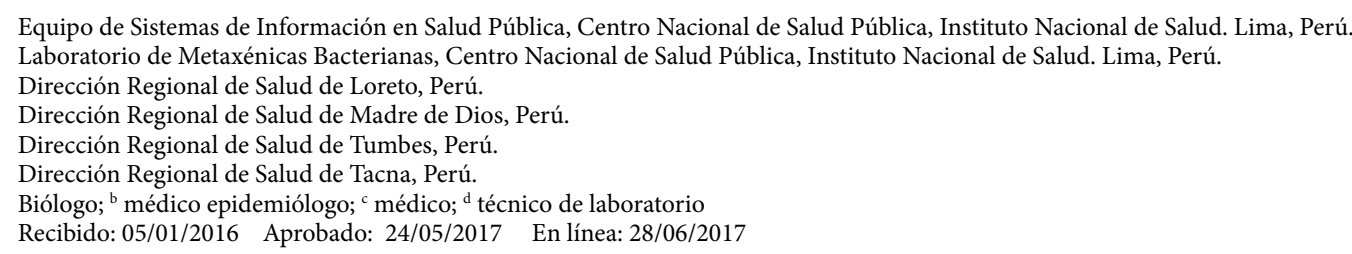


Perú existen antecedentes de estudios en base a encuestas serológicas ${ }^{(6)}$ y de vigilancia nacional de febriles, enfermedades metaxénicas y zoonóticas, de las cuales las rickettsiales son importantes indicadores diferenciales para establecer etiologías, considerando la similar presentación clínica, que podrían resultar determinantes para acciones preventivas y tratamientos oportunos que eviten complicaciones en cuadros clínicos de síndrome febril (7).

La persistencia de anticuerpos después de una infección por Rickettsias es variable, y depende del patógeno involucrado y los factores inherentes al hospedero. Aunque mayoritariamente presentan un curso benigno, una elevada sospecha diagnóstica para el inicio precoz del tratamiento son beneficiosos para la evolución clinica. La etapa aguda, siguiente al contacto con el vector, en la mayor parte de casos, implica básicamente compatibilidad con sindrome febril de caracter no diferenciado, que puede durar entre 7 a 10 dias, generalmente sin complicaciones mayores en individuos inmunocompetentes, muchas veces coincidente con la aparicion de anticuerpos lgM. La fase convaleciente o tardía, que ocurre al menos dos semanas despues, evidencia anticuerpos lgG, que se emplean para evaluar seroconversion comparativamente a un suero obtenido tempranamente, siendo esta la mejor alternativa diagnóstica de la enfermedad, aun cuando existen casos en que la respuesta humoral es más tardía de lo usual ${ }^{(8,9)}$.

La inmunofluorescencia indirecta (IFI) es considerada la técnica de referencia en estudios serológicos para Rickettsia pues presenta una sensibilidad de $91,7 \%$ y una especificidad de $82,0 \%{ }^{(10)}$, valores que pueden variar según los antígenos empleados (11), la oportunidad de obtención de muestras, entre otros factores. Con respecto a la evaluación por serología de Ehrlichia, la sensibilidad para IgG es de $82-100 \%$ y de $27-37 \%$ para $\lg M^{(6,12)}$.

El objetivo del presente estudio fue determinar prevalencia de anticuerpos a Rickettsia y Ehrlichia en localidades de cuatro regiones fronterizas del territorio peruano, con diferentes ecosistemas y tendencias climáticas, Loreto, Madre de Dios, Tumbes y Tacna, a propósito de una encuesta epidemiológica llevada a cabo los años 2010 y $2011^{(13)}$, considerando que diversos reportes ${ }^{(5,6,12)}$ sustentan una circulación de anticuerpos para este grupo de enfermedades infecciosas, con características de infección latente o tardía con carácter endémico.

La motivación principal al realizar estudios en fronteras, radica que, al constituir escenarios, dinámicos, con desplazamientos continuos, se observa globalización y biodiversidad (14), convirtiéndose en ambientes ecoepidemiológicos sui generis para la circulación de microorganismos con las características de Rickettsia y Ehrlichia.

\section{MENSAJES CLAVE}

Motivación para realizar el estudio. La enfermedad febri inespecífica incluye agentes como Rickettsias y Ehrlichias, que en Perú son temas de salud pública poco estudiados, especialmente en los departamentos fronterizos del país.

Principales hallazgos. Se analizaron 1634 personas de Loreto, Madre de Dios, Tumbes y Tacna, evidenciándose una prevalencia de contacto reciente de 10,6 y 3,7\% para Rickettsias y Ehrlichias respectivamente y de, 23,3 y $19,0 \%$ de contacto pasado para Rickettsias y Ehrlichias respectivamente, indicando el carácter endémico de ambas infecciones.

Implicancias. La circulación de Rickettsias y Ehrlichias plantea la necesidad de implementar vigilancia activa de enfermedad febril inespecífica y planificar acciones de prevención.

\section{EL ESTUDIO}

\section{DISEÑO}

Estudio transversal en pobladores de zonas fronterizas tropicales (Loreto y Madre de Dios), semitropical (Tumbes) y templada (Tacna). Las entrevistas se realizaron en los meses de abril a junio de 2010 en Loreto y Madre de Dios y, de marzo a mayo de 2011 en Tacna y Tumbes. La actividad económica productiva predominante para las localidades estudiadas fueron el comercio y la agricultura.

\section{POBLACIÓN}

Las características de la población se detallan en un estudio previo ${ }^{(13)}$. Las localidades fueron seleccionadas en coordinación con el área de epidemiología de las direcciones regionales de salud (DIRESA) de cada región, tomando en cuenta los reportes de casos de rickettsiosis a través del sistema nacional de vigilancia de infecciones febriles inespecíficos y enfermedades metaxénicas basadas en laboratorio.

\section{MUESTRA}

El tamaño de muestra determinado en nuestro estudio previo (13) consideró a cada departamento como dominio independiente, estimando prevalencia del $50 \%$, nivel de confianza $95 \%$, error $5 \%$, y posible rechazo de participación del 15\%, resultando una muestra representativa en cada región de 380 personas, correspondiendo a una persona por familia o vivienda. Empleando un mapa de la zona, se seleccionó y zonificó las áreas de muestreo, el cual fue aleatorio, con sorteo de la zona de inicio y sentido de avance horario.

\section{INSTRUMENTO}

Se utilizó una ficha de recolección de datos con preguntas sobre las características demográficas y sobre algunas condiciones de su entorno epidemiológico. 


\section{CRITERIOS DE INCLUSIÓN}

Se incluyeron individuos de diversos grupos de edad, con residencia permanente mínima de un mes, en las localidades de las cuatro regiones y que aceptaron participar después de recibir la información correspondiente al estudio y firma del consentimiento informado.

\section{VARIABLES}

Se obtuvo muestras de una sola persona por familia o vivienda y en la ficha se indicó la procedencia de los sujetos, su edad y sexo.

La presencia de anticuerpos contra antígenos rickettsiales, se define en la prueba IFI como aquella que exhibe positividad reactiva hacia algún antígeno de rickettsias. En general, están establecidos como punto

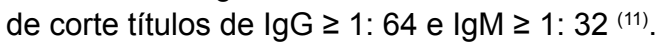

Para el propósito del presente estudio, se hizo la diferenciación entre contacto de infección pasada a toda persona con anticuerpos $\lg G$ en título igual a $1 / 64$ y contacto de infección reciente a toda persona con anticuerpos $\lg$ totales $\mathrm{y} / \mathrm{o} \mathrm{lg}$ en títulos mayores o iguales a $1 / 128$, dado que, en zonas endémicas, la prevalencia de anticuerpos en población sana puede ser elevada.

Los valores de corte, generalmente, son constantes en una misma especie de Rickettsia o especies muy relacionadas, tal como se evidencia en el estudio de Panamá (15); la reacción serológica inició a 1/32, hasta determinar el título final empleando varios antígenos rickettsiales. Se observaron títulos IgM de 1/32 para $R$. amblyommii y $1 / 64$ para $R$. rickettsii, títulos $\lg$ de $1 / 128$ para $R$. amblyommii y $1 / 512$ para $R$. rickettsii. $\mathrm{Al}$ obtener una segunda muestra dos semanas después, se observó elevación de títulos IgG hasta $1 / 512$ para $R$. amblyommii y $1 / 1024$ para $R$. rickettsii, demostrando la seroconversión. En Europa se establece infección con sueros pareados en etapa aguda y convaleciente, los obtenidos en fase inicial con títulos $\lg M>1 / 64$ - IgG>1/128 son sugestivos de infección reciente y por seroconversión con títulos al cuádruple son considerados significativos ${ }^{(11)}$.

Para la presencia de anticuerpos contra antígenos de Ehrlichia a través de la prueba IFI, se estableció el punto de corte de $1 / 50^{(16)}$. Las recomendaciones para ehrlichiosis por el grupo de trabajo en enfoques de consenso incluye títulos $\geq 1 / 256$ y para confirmar la enfermedad títulos de 1/64; sin embargo, los esfuerzos de vigilancia en Perú han considerado los casos por IFI con títulos de $1 / 64$, independiente de la magnitud del título de corte ${ }^{(17) .}$
Por lo tanto, según las consideraciones, para la presencia de anticuerpos a Rickettsia y Ehrlichia, se establecieron títulos $\geq 1 / 128$ como contacto o infección reciente y títulos de 1/64 como contacto o infección pasada.

\section{PROCEDIMIENTO}

Para obtención de sueros se extrajeron 2 a $3 \mathrm{~mL}$ de sangre venosa de los sujetos participantes, en tubos al vacío sin anticoagulante; solo en casos de niños menores de 5 años, fue de 1 a $2 \mathrm{~mL}$. Separado el suero, se transportó al Instituto Nacional de Salud (INS) siguiendo las reglas de bioseguridad y cadena de frio, para preservar la calidad de muestras.

Mediante la prueba de referencia IFI, que provee evidencia indirecta de infección o exposición, al detectar anticuerpos reactivos en el huésped para rickettsias y ehrlichias, se determinó títulos de $\lg$ totales e $\operatorname{lgG}{ }^{(18)}$. Se emplearon láminas in house impregnadas con antígenos de Rickettsia canadensis (grupo ancestral), Rickettsia akari (grupo transicional), filogenéticamente relacionados al grupo tifus y fiebres manchadas, respectivamente, cultivadas en células ATCC Vero y Ehrlichia muris cultivadas en células ATCC DH-82. Se incluyeron controles positivos y negativos comerciales (PanBio) y caracterizados (clínica, epidemiológica y laboratorialmente) y, conjugado antihumano (Sigma Aldrich). El proceso se realizó en nivel II de bioseguridad.

\section{PLAN DE ANÁLISIS}

Se calculó frecuencias y porcentajes para cada una de las variables de interés, prevalencia de anticuerpos para Rickettsia y Ehrlichia en cada región, con sus respectivos intervalos de confianza al 95\% (IC95\%), empleando STATA versión 12.1.

\section{ASPECTOS ÉTICOS}

El estudio contó con aprobación del Comité de Ética del Instituto Nacional de Salud de Perú.

\section{HALLAZGOS}

Se incluyó un total de 1634 individuos, divididos en 424 para la región Tumbes, 388 para Tacna, 410 para Loreto y 412 para Madre de Dios. En nuestra publicación previa se estableció mayor frecuencia del sexo femenino $(65,7 \%)$, y la mediana de edad en 32 años en los sujetos participantes ${ }^{(13)}$.

Se evidenció un total de $551(33,8 \%)$ casos positivos a Rickettsia para Ig totales, de los cuales $172(10,6 \%)$ fueron infecciones recientes (títulos $\geq 1 / 128$ ) y, 379 $(23,3 \%)$ infecciones pasadas (títulos 1/64). Para Ehrlichia 
Tabla 1. Prevalencia de infección reciente y pasada por ehrlichias y rickettsias en pobladores de departamentos fronterizos del Perú

\begin{tabular}{|c|c|c|c|c|c|c|c|c|}
\hline \multirow{3}{*}{ Departamento } & \multicolumn{4}{|c|}{ Presencia de anticuerpos a ehrlichias } & \multicolumn{4}{|c|}{ Presencia de anticuerpos a rickettsias } \\
\hline & \multicolumn{2}{|c|}{$\begin{array}{c}\text { Infección reciente } \\
(\geq 1 / 128)^{*}\end{array}$} & \multicolumn{2}{|c|}{$\begin{array}{c}\text { Infección pasada } \\
1 / 64^{\dagger} \\
\end{array}$} & \multicolumn{2}{|c|}{$\begin{array}{c}\text { Infección reciente } \\
(\geq 1 / 128)^{*}\end{array}$} & \multicolumn{2}{|c|}{$\begin{array}{c}\text { Infección pasada } \\
1 / 64^{\dagger} \\
\end{array}$} \\
\hline & $\mathrm{n}(\%)$ & IC95\% & $\mathrm{n}(\%)$ & IC95\% & $\mathrm{n}(\%)$ & IC95\% & $\mathrm{n}(\%)$ & IC95\% \\
\hline $\begin{array}{l}\text { Loreto } \\
(n=410)\end{array}$ & $10(2,4)$ & $(1,6-3,2)$ & $67(16,3)$ & $(12,7-20,0)$ & $31(7,6)$ & $(4,9-10,1)$ & $58(14,1)$ & $(10,8-17,5)$ \\
\hline $\begin{array}{l}\text { Madre de Dios } \\
(n=412)\end{array}$ & $35(8,5)$ & $(5,7-11,2)$ & $86(20,9)$ & $(17,0-24,9)$ & $39(9,5)$ & $(6,6-12,3)$ & $97(23,5)$ & $(19,4-27,6)$ \\
\hline $\begin{array}{l}\text { Tacna } \\
(\mathrm{n}=388)\end{array}$ & $12(3,1)$ & $(1,8-4,4)$ & $75(19,3)$ & $(15,6-23,1)$ & $62(16,0)$ & $(12,3-19,7)$ & $123(31,7)$ & $(27,1-36,3)$ \\
\hline $\begin{array}{l}\text { Tumbes } \\
(n=424)\end{array}$ & $4(0,9)$ & $(0,5-1,3)$ & $83(19,6)$ & $(16,0-23,3)$ & $40(9,4)$ & $(6,6-12,2)$ & $101(23,8)$ & $(19,7-27,9)$ \\
\hline $\begin{array}{l}\text { Total } \\
(n=1634)\end{array}$ & $61(3,7)$ & $(3,0-4,4)$ & $311(19,0)$ & $(17,1-21,0)$ & $172(10,6)$ & $(9,1-12,1)$ & $379(23,3)$ & $(21,2-25,3)$ \\
\hline
\end{tabular}

*Se determinó contacto de infección reciente a toda persona con anticuerpos lg totales y/o lgG en títulos mayores o iguales a $1 / 128$ medido por inmunofluorescencia indirecta.

†Se determinó contacto de infección pasada a toda persona con anticuerpos IgG en título igual a 1/64 medido por inmunofluorescencia indirecta.

se evidenciaron $372(22,8 \%)$ casos positivos para Ig totales, de los cuales $311(19,0 \%)$ correspondieron a infecciones pasadas y, $61(3,7 \%)$ infecciones recientes, con 35 (8,5\%) casos de ehrlichiosis en Madre de Dios y $62(16,0 \%)$ casos de rickettsiosis en Tacna.

La prevalencia de infección reciente y pasada para ehrlichias fue de 3,7\% (IC95\%: 3,0-4,4) y $19,0 \%$ (IC95: 17,1-21,0) respectivamente, mientras que para rickettsias fue de 10,6\% (IC95\%: 9,1-12,1) y 23,3\% (IC95\%: 21,2-25,3) respectivamente (Tabla 1).

\section{DISCUSIÓN}

El ciclo de vida de las Rickettsia involucra factores ecológicos como clima y vegetación relacionada con la presencia de garrapatas y factores estacionales ${ }^{(3)}$ para determinar la transmisión en humanos. La complejidad de las interacciones entre agente, vector y reservorio con el hombre, hacen que la comprensión integral de la ecoepidemiología de las rickettsiosis requiera de enfoques multidisciplinarios.

Los resultados obtenidos de infección reciente con $3,7 \%$ a Ehrlichia y $10,6 \%$ a Rickettsia, fueron similares a los reportados en Colombia ${ }^{(19)}$ con 3,3\% a Ehrlichia y $7,8 \%$ a Rickettsia, que se explican por la relación con actividades al trabajo en campo, la interacción con animales como probables reservorios y con vectores ${ }^{(13)}$.

Una limitante a considerar en la prueba IgG por IFI en rickettsiosis durante la primera semana de la enfermedad, es su negatividad en aproximadamente el $80 \%$ de los pacientes, por lo que una serología negativa en fase aguda no excluye el diagnóstico (13). En la evaluación de una infección aguda, es estratégico verificar el alza del título de anticuerpos en sueros de fase aguda y convaleciente ${ }^{(8)}$.
Los valores relativamente altos obtenidos para contacto o infección pasada (títulos de 1/64) de 19,0\% para ehrlichias y $23,3 \%$ para rickettsias), estarían indicando un comportamiento endémico en áreas geográficas periurbanas y rurales con características ecoepidemiológicas sui generis, y de alta afluencia poblacional ${ }^{(14)}$ como son las zonas fronterizas estudiadas por primera vez en nuestro país con este propósito.

En Norte América se han presentado brotes de rickettsiosis en localidades fronterizas de México y Estados Unidos (1) con prevalencias entre un 5 a $40 \%$, debido a factores estacionales ${ }^{(3)}$, por lo que es imprescindible tener en cuenta los datos de reactividad basal de la población ${ }^{(20)}$, sobre todo en zonas endémicas. Después de 70 años de ocurrido un brote epidémico de síndrome febril en Colombia, con una letalidad del $95 \%$, se realizó un estudio serológico que evidenció una seroprevalencia de rickettsiosis de $40,3 \%$ en personas sanas ${ }^{(20)}$, hecho que debe ser tomado en consideración al interpretar el silencio epidemiológico que tenemos actualmente en regiones de Perú que, en el siglo pasado sufrieron el embate del tifus epidémico ${ }^{(4)}$.

Concluimos que existe una mayor prevalencia para contacto o infección pasada a rickettsias y ehrlichias en población de localidades fronterizas de cuatro regiones del territorio peruano, lo cual evidencia el comportamiento endémico de ambos agentes infecciosos en dichas áreas geográficas con características ecoepidemiológicas de zonas periurbanas y rurales.

Es necesario complementar la información sobre la cadena epidemiológica de rickettsiosis y ehrlichiosis en Perú, evidenciando la presencia de anticuerpos específicos en animales domésticos, peridomiciliarios y sinantrópicos en cada región, para verificar la condición de 
reservorios, y detectar especies de rickettsias y ehrlichias en ectoparásitos existentes en nuestro territorio, lo cual puede ser motivo de posteriores estudios en el tema.

Agradecimientos: al personal del CIETROP-INS y de los Laboratorios Referenciales Regionales de Loreto, Madre de Dios, Tumbes y Tacna por todo el apoyo brindado.

Contribuciones de autoría: EAR y RPS participaron en la concepción del artículo, la recolección, análisis de datos y su redacción final. Además, EAR gestionó la obtención del financiamiento. Asimismo, el aporte de PM fue con el material de estudio, recolección, análisis e interpretación de los datos. Finalmente, CA, CP, RG y SS, aportaron material de estudio y brindaron asesoría técnica durante la realización del estudio. Todos los autores aprobaron la versión final del artículo.

Fuente de financiamiento: estudio fue financiado por el Instituto Nacional de Salud de Perú.

Conflictos de interés: los autores declaran no tener conflictos de interés.

\section{REFERENCIAS BIBLIOGRÁFICAS}

1. Hidalgo M, Faccini AA, Valbuena G. Rickettsiosis transmitidas por garrapatas en las Américas: avances clínicos y epidemiológicos,y retosen eldiagnóstico. Biomédica;2013;33(1):1-54.

2. Labruna MB. Ecology of rickettsia in South America. Ann N Y Acad Sci. 2009;1166:156-66. doi: 10.1111/j.1749-6632.2009.04516.x.

3. Evia JRB. Fiebre manchada de las Montañas Rocosas. Rev Latinoamer Patol Clin, 2009;56(3):193-208.

4. Calvo Cajigas A, Chevarría Pacheco L, García Uscamayta L, Arizábal Pisco M, QuispeMongeM.Tifusexantemáticoenlas provincias de Quispicanchis, Paucartambo y Acomayo del departamento del Cusco, Perú. Rev Peru Med Exp Salud Pública. 2003; 20(3):145-9.

5. Labruna MB, Mattar $S$, Nava $S$, Bermúdez S, Venzal JM, Dolz G, et al.Rickettsiosis en América Latina, el Caribe, España y Portugal. Rev. MVZ Córdoba. 2011,16(2):2435-57.

6. Moro PL, Shah J, Li O, Gilman RH, Harris N, Moro MH. Short report: serologic evidence of human ehrlichiosis in Peru. Am J Trop Med Hyg. 2009;80(2):242-4.

7. Walker DH. Rickettsiae and rickettsial infections: the current state of knowledge. Clin Infect Dis. 2007; 45Suppl 1:S39-44.

8. Biggs HM, Behravesh CB, Bradley KK, Dahlgren FS, Drexler NA, Dumler S, et al. Diagnosis and management of tickborne rickettsial diseases: Rocky Mountain spotted fever and other spotted fever group rickettsioses, ehrlichioses, and anaplasmosis United States. MMWR Recomm Rep 2016;65(2):1-44. doi: 10.15585/ mmwr.rr6502a1.
9. Monterde-Álvarez ML, Calbet-Ferré C, Rius-Gordillo N, Pujol-Bajador I, Ballester-Bastardie F, Escribano-Subías J. Rickettsiosis tras la picadura de una garrapata: una clínica sutil en muchas ocasiones, debemos estar atentos. Enferm Infecc Microbiol Clin. 2017; 35:100-3.

10. Anaya E, Morón C, Arias P, Chauca J, Román R. Evaluación de pruebas de elisa e inmunofluorescencia indirecta para la detección de anticuerpos IgM contra rickettsiosis. Rev Perú Med Exp Salud Pública. 2008; 25(3):336-9.

11. Oteo JA, Nava S, Sousa RD, Mattar S, Venzal JM, Abarca K, et al. Guías Latinoamericanas de la RIICER para el diagnóstico de las rickettsiosis transmitidas por garrapatas. Rev Chilena Infectol. 2014;31(1):54$65 . \quad$ doi: $10.4067 /$ S071610182014000100009 .

12. Hernández-Ayazo $\mathrm{H}$, Marien-Clarete S. Entendiendo las ehrlichiosis humanas y destacando a un agente causal: Anaplasma phagocytophilum. Rev cienc biomed. 2013;4(1):165-9.

13. Palacios-Salvatierra R, Anaya-Ramírez E, Juscamayta-López J, Cáceres-Rey $\mathrm{O}$, Mendoza-Uribe L, MosqueraVisaloth P, et al. Perfil epidemiológico y molecular de Rickettsiosis en localidades de frontera peruana. Rev Peru Med Exp Salud Pública. 2017;34(1):76-84. doi: 10.17843/ rpmesp.2017.341.2769.

14. Ministerio de Relaciones Exteriores. Desarrollo e Integración fronteriza [Internet]. Lima: Dirección de Desarrollo e Integración Fronteriza; 2012. [citado 22 de diciembre de 2015]. Disponible en: http://www.rree.gob. pe/politicaexterior/Documents/ Propuesta $\% 20$ contenido\% 20
folleto\%20DDF\%20(29MAY12).pdf

15. De Lucas J, García E, García G, Castro A, Lyons C, Bermúdez CSE.

Nuevo caso de rickettsiosis humana en Panamá, a partir de evidencia serológica y clínica. Rev med Panamá, 34:40-3.

16. Paulino R., Analí et al. Detección serológica de Ehrlichia canis y Ehrlichia chaffeensis en personal de clínicas veterinarias en Lima Metropolitana. Rev investig vet Perú. 2013;24(2):217-21.

17. Gómez Muchotrigo BL. Detección de anticuerpos contra Ehrlichia spp. en propietarios de caninos domésticos con ehrlichiosis. [Tesis]. Lima: Universidad Nacional Mayor de San Marcos, Facultad de Medicina Veterinaria; 2014.

18. Paddock CD. Perspectivas sobre el diagnóstico de laboratorio de enfermedades rickettsiales en el siglo 21 . Acta med costarric. 2013;55(3):13-24.

19. Ríos R, Franco $S$, Mattar S, Urrea M, Tique V. Seroprevalencia de Leptospira sp.,Rickettsia sp. y Ehrlichia sp. en trabajadores rurales del departamento de Sucre, Colombia. Infect. 2008;12(2):319-24.

20. Hidalgo M, Sánchez R, Orejuela $L$, Hernández J, Walker DH, Valbuena G. Prevalence of antibodies against spotted fever group Rickettsiae in a rural area of Colombia. Am J Trop Med Hyg. 2007;77:378-80

Correspondencia: Elizabeth Anaya Dirección: Equipo de Sistemas de Información en Salud Pública, Centro Nacional de Salud Pública, Instituto Nacional de Salud. Cápac Yupanqui 1400, Lima 11. Lima, Perú Teléfono: (511) 748-1111 anexo 2169 Correoelectrónico: eanaya@ins.gob.pe 